Mots. Les langages du politique

\title{
La métaphore sportive dans la presse en URSS et en Russie
}

Inna Khmelevskaia

\section{(2) OpenEdition \\ Journals}

Édition électronique

URL : https://journals.openedition.org/mots/1031

DOI : $10.4000 /$ mots. 1031

ISSN : 1960-6001

Éditeur

ENS Éditions

Édition imprimée

Date de publication : 1 juillet 2007

Pagination : 51-63

ISBN : 978-2-84788-112-7

ISSN : 0243-6450

Référence électronique

Inna Khmelevskaia, "La métaphore sportive dans la presse en URSS et en Russie », Mots. Les

langages du politique [En ligne], 84 | 2007, mis en ligne le 01 juillet 2009, consulté le 23 avril 2022. URL : http://journals.openedition.org/mots/1031 ; DOI : https://doi.org/10.4000/mots.1031 


\section{La métaphore sportive dans la presse en URSS et en Russie}

Le sport a toujours présenté un intérêt en tant que source de métaphore, car il «se propose de canaliser ces tensions, particulièrement exacerbées avec le développement du monde industriel, en leur conférant une forme symbolique, quasi rituelle, en les encadrant par des règles et des règlements» (Lejeune, 2001, p. 22).

Si la métaphore sportive est souvent utilisée dans le discours politique, c'est que la politique est souvent associée à une compétition ou un jeu sportif. Tout en admettant que cette association entre sport et politique est universelle, je voudrais relever ici les particularités de l'emploi des emprunts au langage des sports dans le discours politique en Russie, et surtout essayer d'observer le développement dans le temps du recours à ce langage.

Le discours de presse politique est caractérisé par l'intertextualité au même titre que le discours littéraire. Le système des métaphores entre dans l'ensemble des moyens pragmatiques de création du texte. Une métaphore appartient à l'auteur, mais elle correspond à un modèle métaphorique que le public connait bien et qui repose sur un concept familier : "La compréhension concerne des domaines entiers d'expérience et non des concepts isolés» (Lakoff, Johnson, 1999, p. 127). La métaphore est ce par quoi «nous pouvons acquérir quelque chose de neuf» (Aristote, 1991, p. 302), en passant toutefois par quelque chose de familier. Son effet cognitif réside dans sa capacité à redessiner un champ d'expérience à l'aide des termes appartenant à d'autres champs, à d'autres concepts, lesquels doivent à leur tour avoir quelque poids dans la conscience du public. La langue sportive présente un fonds pour la métaphorisation, comme toute sphère d'activité aussi pratiquée, et les concepts d'opposition, de jeu d'équipe, de compétition sont traditionnellement évoqués dans le domaine politique. Sans affirmer que l'usage de la métaphore sportive est une norme pour le discours de presse - s'il convient de parler de norme dans l'emploi de la métaphore -, j'insisterai sur la régularité de sa présence dans les textes journalistiques.

La présence de la métaphore sportive dans ce que Anatoly Tchudinov (qui a consacré plusieurs ouvrages à la conceptualisation métaphorique de la réalité

Université Paris 12 - Val-de-Marne, CEDITEC, innakhmelevskaia@yahoo.fr 
en Russie moderne) appelle «la mosaïque métaphorique» du discours politique (Tchudinov, 2004), hier et aujourd'hui, est régulière sans être dominante. Tout en étant un champ de référence "sans danger» car, à première vue, apolitique, ce champ met à la disposition des politiciens et de la presse des outils de comparaison assez puissants car le sport, une activité facilement appréhendable en tant que fait culturel, véhicule les concepts primordiaux de l'existence : opposition, concurrence, victoire, défaite et même vie et mort.

Deux raisons essentielles président au développement de la métaphore sportive dans les textes de la presse généraliste et, notamment, politique, en Russie :

- le développement du sport en tant que phénomène médiatisé, que spectacle, qui l'amène au rang des concepts universels et conduit à la banalisation et à la popularisation de la langue sportive, de sorte que les métaphores puisées dans cette sphère se rapportent à un concept existant dans la conscience publique - c'est-à-dire de sorte qu'un modèle métaphorique soit possible ;

- le changement intervenu dans le style journalistique, la démocratisation qui a suivi l'écroulement de l'Union soviétique ayant entrainé une déstandardisation de la langue de presse.

Pour mon étude, j'ai choisi des articles tirés des trois titres essentiels de la presse soviétique de l'époque : Pravda [La Vérité], Krasnaya Zvezda [L'Étoile rouge] et Trud [Le Travail] et se rapportant aux années 1960, 1972, 1973, 1986, c'est-à-dire aux trois dernières décennies de l'URSS qui représentent, pour ainsi dire, trois époques: celle du «dégel» (Khrouchev), celle de la stagnation et enfin celle de la perestroïka. Le choix d'analyser des articles postérieurs à 1960 est lié à l'histoire spécifique du sport en Union Soviétique. Du temps de Staline, les sportifs étaient consignés en URSS sans avoir le droit d'en sortir. La Guerre patriotique a porté un coup dur au développement du sport dans ce pays. C'est seulement après la fin de la guerre et la mort de Staline que le sport national a retrouvé son niveau; les sportifs soviétiques ont alors eu accès à l'arène internationale (qu'on se souvienne de la victoire des footballeurs soviétiques à la Coupe d'Europe de football en 1968). Ce n'est qu'à partir de cette époque que le sport, dans le pays, peut être considéré en tant que phénomène social global.

\section{L’emploi de la métaphore sportive en Union soviétique}

En Russie, le sport a toujours été plus qu'une simple activité physique. Il constituait un outil politique, une idéologie, quasiment une religion. Aussi la métaphore sportive a-t-elle toujours été prisée dans le discours des hommes politiques soviétiques. Dans un pays où l'opposition aux pays occidentaux était perçue comme une sorte de compétition et qui se voulait une puissance à l'esprit sain, le sport constituait un vaste cadre culturel : 
Le sport et la politique en Union soviétique ont été inséparables durant les soixantedix ans de l'existence de l'État soviétique. Toute la différence résidait dans le fait que, du temps de Staline, le sport était un produit de consommation interne, car les sportifs soviétiques à l'époque restaient en dehors des arènes internationales. À la mort de Staline, l'URSS a commencé à participer aux tournois mondiaux et européens. Toute victoire était considérée avant tout comme une réussite de l'URSS, et surtout, si cette victoire était remportée sur les pays capitalistes, comme la preuve de la supériorité du système socialiste. ${ }^{1}$ (M. Prozumenschikov, 2004)

La représentation médiatique du sport a beaucoup de points communs avec le reportage de guerre, mais il faut comprendre qu'en URSS, le sport n'était pas seulement une sublimation de la guerre, une métaphore dépourvue de violence, mais une suite naturelle du combat réel. «À l'époque soviétique, on sacrifiait les sportifs comme on sacrifiait des soldats », témoigne l'écrivain Alexandre Niline $^{2}$, auteur de nombreuses biographies de footballeurs soviétiques. Illustrent cette attitude les paroles de la chanson composée pour le film Вратарь [Gardien de but] (1937) par le poète soviétique Lébédev-Koumatch :

Эй, вратарь, готовься к бою

Часовым ты поставлен у ворот!

Ты представь, что за тобою

Полоса пограничная идет!

[Gardien de but, prépare-toi à la bataille

Tu es une sentinelle devant tes buts

Imagine que c'est la frontière de l'État

Qui est tracée derrière toi.]

Ayant observé le rôle du sport dans la société soviétique, en tant qu'activité mais surtout en tant que symbole, on peut s'étonner que l'utilisation de termes sportifs pour créer une image métaphorique du quotidien - y compris la politique - soit assez rare. Les journaux de l'après-guerre soviétique en recèlent peu. Tout d'abord, une métaphore, quelle qu'elle soit, nait d'une comparaison, d'une conceptualisation, voire d'une analyse; son emploi peut mener à une ambiguïté, ce que les propagandistes voulaient éviter à tout prix. Le discours de la presse soviétique était rigide, abondait en clichés; c'était en quelque sorte un système ritualisé, d'une stabilité artificielle. Les thèmes étaient donnés d'avance, les évaluations approuvées officiellement. Il existait toute une phraséologie, un ensemble d'images-clichés sélectionnées au fil du temps. Le texte avait perdu son cacartère pluridimensionnel. La mainmise du Parti sur la presse donnait un style officiel à toutes les productions écrites (Kokorina, 1992 ;

1. M. Prozumenschikov, «Histoire et modernité : Les documents du passé». Propos recueillis par Vladimir Toltz pour Radio Svoboda le 19 juin 2004.

2. Alexandre Niline, "Le sport présente un intérêt en tant que métaphore », interview donnée à l'Observateur littéraire le 23 mai 2005 (propos recueillis par Andrei Miroshkine). 
Baranov, Kazakevitch, 1991). Dans ces conditions, la métaphore, basée, selon Kleiber, sur une "transgression de l'usage ordinaire des termes et combinaisons, en somme un “délit littéral” " ${ }^{3}$, n'était pas toujours la bienvenue.

Les textes des journaux nationaux de l'époque (Pravda, Krasnaya Zvezda, Trud) étaient sérieux, directs, plats, avec un contenu émotionnel assez élémentaire: textes extatiques décrivant les réussites du peuple soviétique dans les champs et les usines; textes accusateurs, franchement agressifs, décrivant les initiatives politiques des pays occidentaux. Pendant les quinze ans qui ont suivi la guerre, le sport occupait dans ces journaux une position plus que modeste : le quart de la dernière page du journal.

Les journalistes soviétiques avaient pour mission de créer une opposition «positif-négatif», d'opposer le Bien - incarné par l'URSS - au Mal représenté parle monde occidental.

La seconde guerre mondiale a marqué les esprits des Soviétiques. Rarement un journal paraissait sans un reportage consacré à la «lutte du peuple soviétique contre le fascisme » et aux héros de cette lutte; la métaphore militaire, omniprésente, pénétrait tous les textes, y compris les rares articles consacrés au sport et qui portaient des titres comme «Préparons-nous aux batailles footballistiques» (Krasnaya Zvezda $\mathrm{n}^{\circ}$ 19, 23 janvier 1960) ou «Nous ne céderons pas les frontières conquises » (Krasnaya Zvezda $\mathrm{n}^{\circ} 6,5$ janvier 1960).

Il ne faut pas oublier que même si «à l'époque où le football était un jeu d'élite, les footballeurs étaient des héros populaires, héros nationaux, presque comme les premiers aviateurs», selon le témoignage de Niline, le football et le sport en général n'étaient pas considérés comme une activité «sérieuse »; en effet, "le milieu des activités physiques et sportives a souvent souffert d'être mis sous tutelle, voire “infantilisé”", estime Pierre Parlebas (1996). Un milieu qui servait bien la propagande mais dont l'activité - ironie du sort - était conçue comme essentiellement ludique, comme un loisir, et ne pouvant aucunement contribuer à conceptualiser l'expérience de sphères aussi importantes que la politique et l'industrie. Ainsi retrouve-t-on dans les journaux des années soixante la métaphore militaire et agricole, mais très rarement sportive.

Il existe cependant des exceptions, comme les métaphores qui ont été construites avec les mots de la compétition car la vie des Soviétiques était une compétition sur tous les fronts. On se souvient du fameux slogan «Rattrapons et devançons l'Amérique» (titre d'une rubrique de Krasnaya Zvezda enregistrant les progrès de l'industrie soviétique). On évoque la compétition interne, «socialiste» (соцсоревнование), entre les différents secteurs de l'industrie, les usines, etc. (Pravda publie un «Journal de la compétition»), mais aussi un concours avec le monde extérieur, qu'il s'agit de devancer en économie ou - plus tard - dans la course aux armements et les réussites spatiales : «Dans

3. Cité d'après Charbonnel, 1999, p. 102. 
cette compétition pacifique, la victoire sera à nous» (Krasnaya Zvezda nº 13, 16 octobre 1960)

Quant à la métaphore renvoyant au jeu sportif, elle est évoquée assez rarement à l'époque (la télévision en Russie faisait ses premiers pas et le jeu sportif ne bénéficiait pas encore de ce média).

Le style des journaux des années soixante-dix semble moins rigide : nombre de métaphores apparaissent dans les titres. Le sport commence à occuper une place honorable dans la presse. Néanmoins, la métaphore sportive reste rare. Celles qui sont employées deviennent, une fois introduites (et autorisées), des clichés qui enrichissent encore la langue de bois ${ }^{4}$. Ainsi retrouve-t-on le concept de compétition ("Quartier général de la compétition », Trud n²52, 26 octobre 1972) pour un article consacré à la construction industrielle, sans parler des expressions déjà enracinées dans la langue. Employée pour désigner les «exploits» d'ouvriers agricoles et d'usines, la métaphore sportive enrichit les titres: "Tournoi ${ }^{5}$ aux machines » (Trud n 247, 20 octobre 1972 - il s'agit d'un concours d'ouvriers), "Assaut aux buts zeyens» (Trud n² 237, 8 octobre 1972 - article relatant la construction d'une digue à la centrale de Zeya), etc. La métaphore sportive ne se distingue pas par sa diversité et on ne relève pas de textes construits, comme on en verra plus tard, autour d'elle.

À cette étape, il est difficile pour le linguiste de définir si, dans tel ou tel cas, on a eu recours à une métaphore sportive ou à une métaphore militaire, et cela pour de bonnes raisons. La langue sportive et ses moyens expressifs, à l'époque, sont encore en voie de développement. Des mots et expressions que l'on a l'habitude, aujourd'hui, d'entendre dans la bouche d'un reporter sportif s'emploient alors au sens propre. La médiatisation du sport à cette époque n'a pas la même ampleur qu'aujourd'hui ; les premiers reporters sont appelés à rendre, de façon précise et détaillée, le contenu du match pour les supporters qui ne peuvent pas y assister directement; ils remplissent une fonction d'intermédiaire en étant «les yeux» du public accroché à son poste de radio. Il semblerait que la guerre, dans les années cinquante-soixante, ne soit pas assez désémantisée pour que le sport puisse s’exprimer légitimement avec ses termes;

4. I. Lysakova, dans une étude sociologique de la presse soviétique, définit la notion de «langue de bois»: une langue délibérément appauvrie, «non à cause d’une mauvaise prestation des journalistes, mais du caractère totalitaire du système politique», quand "une censure idéologique sévère amène à la prédominance du standard sur l'expressivité dans tous les types d'ouvrages»; une langue aux intonations impératives reflétant le style de communication bureaucratique (1993, p. 83-86).

5. Des termes comme compétition et tournoi ne se perçoivent peut-être pas au premier regard comme des termes sportifs, car ils font partie aujourd'hui du lexique quotidien. Rappelons néanmoins que ces notions sont à l'origine même du sport : les premières manifestations sportives étaient des compétitions et des tournois. Ces termes n'ont d'ailleurs pas perdu leur contexte spécialisé, un tournoi de chevalerie devenant un tournoi des six nations. Je crois légitime d'affirmer qu'en employant ces termes dans le discours politique, le journaliste pense avant tout au sport. 
mais en réalité, le sport continue à puiser dans la sphère de conceptualisation militaire et guerrière. Un échange constant a lieu entre la langue de la guerre et celle du sport, échange à sens unique grâce auquel, aujourd'hui, le match est décrit en termes militaires. Ainsi, dans des contextes identiques, là où aujourd'hui on verrait une association avec un jeu sportif, le reporter paraitrait avoir emprunté à la langue militaire :

Large victoire de la politique de paix (Pravda $\mathrm{n}^{\circ} 301,27$ octobre 1960.)

Une grève totale de quarante-huit heures, qui a eu lieu à la fin février, a été la première contre-attaque de la classe ouvrière dirigée contre la bourgeoisie. ( $\operatorname{Tr} u d \mathrm{n}^{\circ} 237$, 6 octobre 1960.)

Les textes des années quatre-vingt (surtout après 1985, l'arrivée de Gorbatchev et le début de la perestroïka) semblent avoir radicalement changé par rapport à ceux des années antérieures. Ils créent l'illusion d'une certaine liberté de parole. Les articles deviennent analytiques, prennent une tonalité parfois critique envers le pouvoir, facilitée par la proclamation de la glasnost [transparence]. Mais la métaphore sportive est toujours utilisée très modestement et les vieilles formules reviennent.

J'ai pu également faire une curieuse observation : de tous les contextes où j'ai repéré une métaphore sportive, $60 \%$ se rapportent aux rubriques traitant de politique internationale. Le concept de jeu (jeu dangereux, sale jeu) y est très prisé :

Il faut dire franchement et sincèrement que c'est un jeu très dangereux - et dangereux, au premier abord, pour le Japon lui-même. " (sténogramme du discours de Khrouchev du 26 février 1960 dans Krasnaya Zvezda.)

Jusqu'à aujourd'hui, cela [Nuremberg] reste comme un os à travers la gorge pour les cercles revanchards encore vivants qui, d'une façon ou d'une autre, rêvent de «rejouer» les résultats de la deuxième guerre mondiale et du développement de l'Europe d'après-guerre. (Pravda n $274,1^{\text {er }}$ octobre 1986.)

On pourra encore citer les titres suivants :

Round difficile à Paris (Trud n² 247, 20 octobre 1972.)

Sur la ligne d'arrivée du marathon électoral (Trud $\mathrm{n}^{\circ} 261,5$ octobre 1973.)

USA-Japon : jeux de provocation (Pravda $\mathrm{n}^{\circ} 301,28$ octobre 1986)

Joueurs d'atome (Pravda ${ }^{\circ} 299,25$ octobre 1960)

Citons, pour finir sur ce point, cette remarque de A. Tchudinov :

La majorité absolue des métaphores liées aux concepts de jeu et de sport possèdent un potentiel pragmatique négatif dû à la spécificité de la sphère [...] Le jeu est perçu dès le début comme une imitation de l'activité [...] Dans la mentalité russe, on considère le jeu comme un loisir, et non comme une activité sérieuse à laquelle il faut consacrer l'essentiel du temps. 
Sans doute ce type de métaphore était-il employé dans la description de la politique internationale afin de « souligner le caractère secondaire des événements, le manque de sincérité des agents d'action politique» (Tchudinov, 2005).

\section{La métaphore sportive dans le discours politique moderne}

Le discours politique en Russie est un phénomène en voie de développement. La dernière décennie voit apparaitre des modèles métaphoriques passant par des vecteurs conceptuels évoquant la violence, l'agression, la concurrence. (Tchokhonelidze, 2004, p. 1) Ces modèles, à caractère essentiellement émotif, sont destinés à transposer l'attitude appréciative du concept-source à la signification métaphorique.

Le discours politique russe d'aujourd'hui se distingue par un renouvellement fondamental du contenu et de la forme de l'activité communicative, par l'aspiration à un style individuel (importance du «label», de la «marque»), à l'expressivité, mais aussi à une vivacité à la limite du carnavalesque, une décontraction à la limite du dévergondage et de la muflerie politique. La spécificité de ce discours est, dans une grande mesure, conditionnée par les vecteurs conceptuels caractéristiques de la conscience nationale - angoisse, suspicion, manque de confiance, agressivité -, mais aussi par le sentiment de l'incongruité de la situation actuelle, l'absence de repères idéologiques et d'une «idée nationale » qui pourrait unir la société (Tchudinov, 2005).

Pour constituer le corpus contemporain de ma recherche, j'ai pris en compte aussi bien les journaux sur papier qu'en ligne. Je me suis avant tout intéressée aux quotidiens modernes légitimes comme Izvestia (230000 exemplaires), Rossiyskaia Gazeta (400 000), Novaia Gazeta (172 000), aux portails d'information fréquemment consultés sur Internet (Vesti segodnia, Gazeta.ru, Rossiyskie Vestii, Reporter ${ }^{6}$ ) ainsi qu'aux éditions régionales et indépendantes (Lebed, Arabeski, Russian-bazaar, Ai Regnum, Expert Severo-Zapada7, etc., cela pour présenter une mosaïque représentative des textes journalistiques de la Russie d'aujourd'hui.

Après avoir étudié des exemples de métaphore sportive dans le discours politique actuel, je suis arrivée aux conclusions qui suivent.

Il faut tout d'abord noter que la métaphore footballistique domine, devançant même celle qui renvoie au hockey. Ce sport a pourtant acquis en Russie une envergure nationale une dimension mythique qui est celle de l'URSS, de la grande puissance qui a su s'imposer sur l'arène politique internationale ainsi que sur la glace. Le nombre de métaphores renvoyant à ce sport dans notre

6. Respectivement: [http://www] gazeta.lenta.ru, rosvesty.ru, novy.tv/reporter/

7. Respectivement: [http://www] lebed.com, arabeski.globalrus.ru, russian-bazaar.com, regnum. ru, kadis.ru 
corpus est cependant limité. Remarquons que le football s'inscrivait parfaitement dans la doctrine idéologique soviétique qui plaçait le collectif au-dessus de l'individuel. On pourrait dire que le football est un sport prototypique, un sport métaphorisable par excellence.

La métaphorisation du discours politique russe subit aujourd'hui une forte influence anglo-américaine. Ce discours change : les journalistes, à la recherche d'un nouveau style, se tournent vers l'Occident et empruntent certaines figures de style à la presse étrangère. La métaphore sportive véhiculant des notions politiques est notamment répandue aux États-Unis. Les sports nationaux dont les termes deviennent un savoir partagé pour la majorité des locuteurs sont différents en Russie et en Amérique. Cette influence ne se perçoit pas autant dans la métaphore liée, par exemple, à un sport précis - même si, parfois, elle se perçoit dans l'utilisation de termes sportifs purement «américains » : "Qui est à la première base?» (Novaya Gazeta $n^{\circ} 83$, 2-5 novembre 2006). Au contraire, les métaphores qui se rapportent aux notions de compétition, jeu d'équipe, viennent souvent de l'américain, comme des constructions du type «Le favori de la course présidentielle essuie une défaite sur la ligne de départ » (Izvestia ${ }^{\circ}{ }_{20}$, 3 février 2000).

Dans la presse d'aujourd'hui, la métaphore sportive est plus diversifiée que dans les journaux soviétiques. Il subsiste cependant quelques clichés, des expressions standardisées dont abusent les reporters: ainsi, la course présidentielle, la course électorale ou le pressing des autorités ont perdu depuis longtemps toute originalité, de même que la compétition socialiste ou la course aux armements soviétiques. Pourtant, la métaphore sportive évolue et s'enrichit. On relève par exemple l'utilisation d'une expression figée, d'une unité de type proverbial (Khmelevskaia, 2006, p. 262) propre à un sport donné, qui est par elle-même un énoncé culturel et fait référence à un trésor partagé par une communauté - ici, une communauté sportive: "Nous ne voulons pas de ce hockey-là. Pourquoi les élections n'ont-elles pas réussi à se terminer en un seul round? Uniquement pour la raison que la lutte revêtait - et c'est peu dire un caractère antisportif» (Leninskaya smena $n^{\circ} 42,20-26$ octobre 2005). «À six heures et demie du soir, les chiffres apparus sur le tableau du Parlement ont, semble-t-il, plongé l'assistance dans un état de choc : la réforme politique a été soutenue par 294 députés. Il ne leur manquait que six mandats, mais, comme disent les footballeurs, si c'est dans la transversale ou dans les tribunes, c'est pareil » (Delovaya stolitsa $\mathrm{n}^{\circ}$ 15, 12 avril 2004). Ici, l'auteur de l'article fait plus qu'utiliser un terme sportif pour désigner une réalité politique; il essaie de superposer deux espaces culturels habituellement non superposables et, de cette façon, crée une sorte de métaphore générale.

On notera aussi l'emploi, dans le titre ou dans le texte, d'une métaphore générale, ou la construction d'un texte ou d'une partie du texte autour de la métaphore sportive; la construction d’une «métaphore conceptuelle 
développée» qui régit le texte (Tchudinov, 2004) comme, par exemple, dans ces deux extraits :

Les élections au Parlement, c'est le championnat d'une ligue professionnelle, non pas de hockey, mais de politique. Qui remporte la victoire au championnat de hockey? Celui qui a les meilleurs joueurs, des crosses et des patins professionnels [...] On peut dire que c'est le capitaine qui est responsable de la défaite de son équipe, mais ce n'est qu'une partie de la vérité. Même si le capitaine se trompe, le conseil de l'équipe, sous forme du bureau du parti, et un conseil élargi, qui inclut les chefs des supporters, le conseil central, doivent le corriger. (Yabloko Rossii ${ }^{\circ} 5$, 5 février 2000.)

Medvedtchuk est persuadé qu'il n'a pas le droit de céder sur son terrain. Yuschenko est convaincu d'avoir tous les droits à une victoire à l'extérieur. D'autant plus que l'enquête réalisée par quatre instituts montre que la majorité des habitants locaux supportent son équipe. Dans des matches pareils, les compromis sont impossibles. Dans des jeux pareils, comme disent les footballeurs, seul un jeu très dur est possible. (Zerkalo Nedeli $\mathrm{n}^{\circ}$ 16, 24-29 avril 2004.)

En ce qui concerne les concepts essentiels exprimés par la métaphore sportive, les plus réguliers sont ceux d'agression et de pression, de règles du jeu et d'infraction aux règles, de compétition, de jeu d'équipe.

\section{L'agression}

En analysant le contenu de la métaphore, on constate que l'auteur du texte fait volontiers appel à la métaphore sportive quand il y a opposition, voire agression. Il serait excessif d'affirmer que la métaphore sportive est toujours employée avec une connotation négative. Pourtant, les exemples que nous avons relevés montrent que le champ de référence «sport» fait naitre dans le discours politique principalement des métaphores de l'agression : contact brutal, pressing sur tout le terrain, réponse du berger à la bergère, match de mort, etc. :

Angela Merkel est une personne étonnante. Ce qui étonne, c'est son ascension au sein du parti, où elle choisissait les moments propices pour «se démarquer », comme disent les sportifs, d'un concurrent masculin (Radio Svoboda, 2 septembre 2006.)

Ainsi, l'Ouvrier de Nijni déclare que la visite s'est transformée en un véritable show. Le séjour, selon l'idée des organisateurs, devait être une visite non officielle mais amicale, mais l'entretien avec les habitants de Nijni est devenu, comme disent les sportifs, une réponse du berger à la bergère. (Ai Regnum, $1^{\text {er }}$ décembre 2005.)

Ne serait-ce pas dans l'intérêt du Premier ministre d'exciter Medvedtchuk contre Yuschenko, de provoquer une charge brutale entre Notre Ukraine et le SPDU? (Ibid.)

La métaphore sportive et la métaphore militaire se croisent souvent, les deux sphères - celle du sport et celle de la guerre - étant assez proches dans la 
réalité. Le modèle métaphorique «terrain de sport - terrain politique - terrain de bataille » n'a rien perdu aujourd'hui de son actualité. Mais si, dans les journaux soviétiques, on a souvent décrit le sport en termes de guerre, aujourd'hui, ce processus est devenu réciproque. Le sport apparait comme une «forme d'édulcoration progressive de la violence »; on peut même se demander si les reporters ne choisissent pas la métaphore sportive pour banaliser la guerre, pour lui ôter une partie de sa gravité.

Eh bien, comme disent les footballeurs, si tu ne marques pas, tu encaisses. Comme le 11 septembre. (Lebed $\mathrm{n}^{\circ} 237,16$ septembre 2001.)

À la pointe de l'attaque économique russe, comme on s'y attendait, s'est trouvée l'Allemagne, un pays qui, pour la Russie, devient une sorte de Virgile, son guide dans l’Europe. (Cascade ${ }^{\circ}$ 266, 21 juillet-11 aout 2006.)

Le Hamas peut attendre, en prenant un time-out avant le départ. Aujourd'hui, il rappelle un avant-centre qui était assis sur le banc des remplaçants en attendant son heure» (Arabeski, 17 novembre 2004.)

Plus effrayant, plus fort, plus douloureux ${ }^{8}$ : Le terrorisme international a ses propres jeux et ses propres règles. (Rossiyskaya Gazeta ${ }^{\circ}{ }^{147,} 8$ juillet 2005.)

Mobilisation des «demis» [en parlant des officiers de réserve] (Izvestia $n^{\circ} 20$, 3 février 2000.)

J'ai relevé également une association assez typique de la Russie: pouvoirpression, agression envers le peuple. La métaphore banalisée de «pressing du pouvoir » est très fréquente de nos jours dans la presse. La conscience nationale russe considère traditionnellement les autorités, quelles qu'elles soient, comme un agent de pression, une menace potentielle. Cette vision du pouvoir se traduit aussi par la métaphore sportive :

On connaît la chanson : le pouvoir aime, comme jadis, qu'on le remercie avec des larmes et, comme jadis, ne supporte pas qu'on le critique. Et il appliquera à tout mécontent, comme disent les sportifs et les kolkhoziens, un pressing sur tout le terrain. (Novaya Gazeta $\mathrm{n}^{\circ}$ 31, 28 avril 2005.)

Quant au pouvoir, n'oubliez pas qu'il agit toujours selon le principe divide et impera : il fait des avances, soutient, propose des postes et parfois, tout simplement, incarcère. Il sait poursuivre et faire du pressing. (Ai Regnum, 26 mars 2004.)

\section{Les agents du pouvoir politique comme joueurs d'une équipe}

Une autre association est assez régulièrement utilisée : «agents du pouvoir joueurs d'une équipe » (souvent l'équipe nationale) :

8. Le titre est calqué sur le slogan olympique. 
Le banc des remplaçants du nouveau pouvoir: les mêmes visages, mais de profil. Banc des remplaçants : les islamistes palestiniens attendent leur heure. (Arabeski, 17 novembre 2004.)

"Notre Ukraine» fera entrer dans le jeu les personnes qui étaient longtemps restées sur le banc des remplaçants. (LIGA Buisinessinform, $1^{\text {er février 2005.) }}$

Si l'on parle en termes sportifs, ce n'est pas l'État qui joue sur le terrain, mais l'arbitre. C'est l'État qui donne les règles et résout les problèmes stratégiques. (Ibid., 16 janvier 2006.)

C'est l'équipe qui fait le jeu. [titre de l'article consacré au parti] (Rossiyskaia Gazeta n²44, 20 décembre 1997.)

\section{La conceptualisation de la concurrence}

Le pluripartisme et les élections libres dans la nouvelle Russie d'après 1991 ont renforcé la notion de concurrence en faisant se développer la métaphore de la compétition. On peut d'ailleurs se demander si les expressions toutes faites telles que «la course à l'électeur» gardent encore leur caractère métaphorique. Comme il a déjà été mentionné, il semble que le modèle métaphorique de concurrence ait été en grande partie copié sur celui des États-Unis.

C'est ce qui peut provoquer des complications, car la course présidentielle battra son plein et réunir deux-tiers des voix des députés sera beaucoup plus difficile qu'aujourd'hui. [...]

II reste encore assez de temps avant les élections de 2007, et nombreux sont ceux qui craignent sans doute un faux départ, comme disent les sportifs. (Rossiyskie Vesti $\mathrm{n}^{\circ} 20,8-15$ juin 2005.)

Merci à Kerry pour être revenu au score et avoir gagné dans les débats télévisés et pour avoir fait de façon que rendu le match présidentiel passionnant, car ici, les deux adversaires se valent (je parle de leur forme sportive). (Russian-bazaar, 16 janvier 2004.)

«Nous voulons unir nos efforts et aider à rétablir la paix aux Balkans», a déclaré aux journalistes le président de la Finlande. Selon son expression, les pourparlers d'aujourd'hui, c'est un match préliminaire, si on s'exprime en termes sportifs. (Gazeta.ru ${ }^{\circ}$ 4, 21 mai 1999.)

\section{Les règles du jeu et le jeu irrégulier des politiciens}

À l'époque de l'URSS, toute action politique entreprise par le gouvernement était supposée être honnête et juste. La métaphore sportive traduisant une infraction aux règles était donc principalement réservée à la politique des pays ennemis. À présent, Aujourd'hui, la presse l'utilise largement à propos des politiciens du pays : 
Jeu irrégulier de l'équipe de Riabchenko sur le terrain électoral. Jeux sales dans un district paisible.

(Vesti segodnia, 18 février 2006.)

La première motion de censure en Europe serait un sale jeu? (Reporter, 12 mai 2006.) Bien que «jouant la montée», pour employer un terme sportif, les leaders de l'Europe comprennent qu'ils n'ont pas beaucoup de temps. (Ibid.)

Carton jaune à «Sibneft». (Izvestia $\mathrm{n}^{\circ} 213,16$ novembre 2004.)

Le développement de la métaphore sportive dans la presse politique russe s'est produit dans les années 1990-2000. Auparavant, dans une presse contrôlée par le gouvernement soviétique, certaines des métaphores qui apparaissaient étaient récupérées et allaient s'enraciner dans la langue de bois. Une véritable création langagière était difficilement possible à cette époque.

L'apparition d'un style plus fleuri dans la presse russe après 1991 représente, du point de vue linguistique comme du point de vue éthique, un phénomène ambigu. Qu'une métaphore soit ou non appropriée, le langage du sport, même s'il n'est pas employé aussi largement qu'aux États-Unis, participe de façon régulière à la métaphorisation de la réalité - politique notamment. Parmi les sphères de conceptualisation qui enrichissent le plus la métaphore sportive en contexte politique, on a noté agression et pression, règles du jeu et infraction aux règles, compétition, jeu d'équipe. On a souligné que la mise en équivalence courante «le sport est une guerre » s'est inversée : aujourd'hui, « la guerre est un sport».

Quant au but, on dira qu'il traduit, pour le journaliste de presse russe, la vision qu'a le peuple du pouvoir en place : absence totale de confiance dans le processus politique, perçu comme aussi imprévisible et aléatoire que le déroulement d'un match de football; intérêt superficiel pour les jeux électoraux; doute quant à la valeur du travail d'équipe et au respect des règles par les autorités.

\section{Références}

ARISTOTE, 1998 [vers 329 av. J.-C.], Rhétorique, Paris, Gallimard (Tel). традиции

БАРАНОВ А. Н., КАЗАКЕВИЧЕ. Г.,1991, Парламентские дебаты: традиции и новации,

Современный политический язык (От ритуала к метафоре), М., Знание, $\mathrm{n}^{\circ} 10$, стр. 60-63.

[BARANOVA. N., KAZAKEVITCHE. G., 1991, «Débats parlementaires : traditions et innovations. La langue politique contemporaine (du rituel à la métaphore) », Moscou, Znanie, $\mathrm{n}^{\circ} 10$, p. 60-63.] 
CHARBONNEL N., KLEIBER G., 1999, La métaphore entre philosophie et rhétorique, Paris, PUF, $245 \mathrm{p}$.

КАКОРИНАЕ.В., 1992, Стилистические изменения в языке газеты новейшего времени, М.

[KAKORINA E.V., 1992, Changements stylistiques dans la langue de la presse contemporaine, Moscou.]

KHMELEVSKAIA I., 2006, Étude comparative des termes des jeux sportifs en français et en russe et composition du dictionnaire franco-russe, thèse de doctorat, Université Paris 12 Val-de-Marne, $415 \mathrm{p}$.

LAKOFF G., JOHNSON M., 1985, Les métaphores dans la vie quotidienne, Paris, Minuit (Propositions), $250 \mathrm{p}$.

LEJEUNE D., 2001, Histoire du sport (XIXe-XXe siècle), Paris, Christian (Vivre l'histoire), $219 \mathrm{p}$.

ЛЫСАКОВА И.П.,1993, Пресса перестройки, СПб.

[LYSAKOVA I. P., 1993, La presse de la perestroïka, Saint-Pétersbourg.]

чОХОНЕЛИДЗ Н.С., 2004, «Оценочность как основное свойство политического дискурса », Сайт Славянского научно-просветительского центра.

http://www.slavcenter.ge/art/?p=20041216-225820

[TCHOKHONELIDZE N.S., 2004, "Le pouvoir appréciatif comme propriété essentielle du discours politique», Site du Centre d'études slaves, http://www.slavcenter.ge/ art $/ p=20041216-225820]$

чУдИнОВ А. П., 2001, Россия в метафорическом зеркале : когнитивноеисследова ние политической метафоры (1991-2000), Екатеринбург, 238 с.

http://www.philology.ru/linguistics2/chudinov-01.htm

- 2003, «Метафорическая мозачка в современной политической коммуникации », Екатеринбург, 248 c .

http://www.philology.ru/linguistics2/chudinov-оза.htm (глава 1-2)

[TCHOUDINOV A.P., 2001, La Russie au miroir de la métaphore: étude cognitive de la métaphore politique (1991-2000), Ekaterinbourg, 238 p. http://www.philology.ru/ linguistics2/chudinov-01.htm

-2003, «La mosaïque métaphorique dans la communication politique contemporaine », Ekaterinbourg, $248 \mathrm{p}$.

http://www.philology.ru/linguistics2/chudinov-03a.htm (chapitres 1-2)] 